Letter to the Editor

\title{
Longitudinal evaluation of posterior corneal changes after LASIK in high myopia: a swept-source optical coherence tomography study
}

\author{
Kelvin H Wan MRCSEd, ${ }^{1}$ Tommy CY Chan FRCS, ${ }^{1}$ Marco Yu PhD ${ }^{1}$ and Vishal Jhanji MD \\ FRCOphth $^{1,2}$
}
1. Department of Ophthalmology and Visual Sciences, The Chinese University of Hong Kong, Hong Kong, China
2. Department of Ophthalmology, University of Pittsburgh School of Medicine, Pittsburgh, PA, USA

Correspondence: Tommy CY Chan, Department of Ophthalmology and Visual Sciences, the Chinese University of Hong Kong, Hong Kong, China E-mail: tommychan.me@gmail.com

Received 12 February 2018; accepted 18 February 2018

Conflict of interest: None

Funding sources: None

This is the author manuscript accepted for publication and has undergone full peer review but has not been through the copyediting, typesetting, pagination and proofreading process, which may lead to differences between this version and the Version of Record. Please cite this article as doi: $10.1111 /$ ceo.13178

This article is protected by copyright. All rights reserved. 
LASIK intends to decrease the corneal power by increasing the radius of corneal curvature in myopic correction. Though it does not directly affect the posterior corneal surface, modification is not unexpected since LASIK can weaken the cornea especially in high myopic correction. High myopia poses a significant risk for the development of regression and ectasia after LASIK, $^{1}$ detection of these conditions requires knowledge about the posterior corneal curvature. Owning to the faster scanning time and longer wavelength, swept-source optical coherence tomography (SS-OCT) offers a higher reliability in post-LASIK corneal measurements as compared to Scheimpflug imaging. ${ }^{2}$ It remains controversial how the posterior cornea would behave after LASIK over time. We hypothesize that by evaluating the high myopic eyes ( $\geq-5$ diopters, $D$ ), this cohort would allow corneal changes, if any, to be detected earlier with greater sensitivity. We evaluated the longitudinal posterior corneal changes over 3 years using SS-OCT after high myopic LASIK correction, this series has the longest follow-up with longitudinal measurements obtained from a SS-OCT.

Details of our surgical technique and SS-OCT imaging have been described. ${ }^{3}$ Briefly, superior hinge LASIK flaps were created using a 150-kHz IntraLase femtosecond laser (Abbott Medical Optics, Chicago, IL) with intended thickness and flap diameter

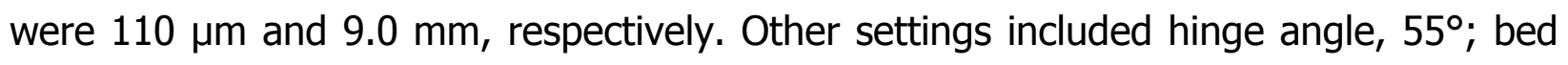
energy, $0.75 \mathrm{~mJ}$; spot separation, $6 \mathrm{~mm}$; line separation, $6 \mathrm{~mm}$; side-cut energy, 1.1 $\mathrm{mJ}$; pocket width, $200 \mathrm{~mm}$; pocket start depth, $210 \mathrm{~mm}$; and both pocket tangent and radian spot separation, $4 \mathrm{~mm}$. Stromal ablation was performed with Allegretto Wave \& Eye-Q 400-Hz laser (Wavelight Laser Technologie AG, Germany) using a $6.5 \mathrm{~mm}$ optical zone. The keratometry and topography data were obtained from the TopoPachy-Map scan protocol on a SS-OCT (Casia SS-1000; Tomey, Nagoya, Japan). We evaluated the posterior corneal changes including: the radius of an $8 \mathrm{~mm}$ best-fit sphere (BFS), mean keratometry, and corneal astigmatism (converted to power vector 
components $\mathrm{J0}$ and J45). ${ }^{4}$ We used one-way analysis of variance model with repeated measures to compare the differences in these parameters at different time points (preoperative, month 3, year 3).

71 eyes from 41 patients with a preoperative mean spherical equivalent (SEQ) of $-6.67 \pm 1.34 \mathrm{D}$ (range: -5.00 to $-11.00 \mathrm{D}$ ) were included. After LASIK, posterior mean keratometry decreased from $-5.92 \pm 2.01 \mathrm{D}$ preoperatively to $-5.97 \pm 2.01 \mathrm{D}$ at 3 months $(p<0.001)$ which remained stable at 3 years $(p=0.145)$. Significant differences in the radius of the BFS at the posterior cornea were found between baseline and 3 months $(p<0.001)$ but not from baseline to year $3(p=0.281)$. Time course changes in $J 0$ and J45 were not statistically significant across any comparisons $(p \geq 0.260)$. SEQ and uncorrected distant visual acuity remained stable from 3 months onwards ( $p \geq 0.073$ ). For completeness, the postoperative stability in the anterior mean keratometry paralleled the posterior mean keratometry (Table 1).

We previously reported a forward protrusion on the posterior cornea within the first postoperative year after LASIK, $^{3}$ this is in agreement with the statistically significant increase of the negative central power of the posterior cornea observed at 3 months postoperatively in this study, which remained stable afterwards. The radius of the BFS was steepened at an early stage but then returned to baseline 3 years after LASIK in the current study. We speculate these time dependent changes reflect the wound healing processes after LASIK, where active wound healing occurs up to 6 months postoperatively, followed by wound remodelling up to 3 years. ${ }^{5}$ Circumferential severing of the corneal lamella weakened the corneal biomechanics, resulting in the steepening of the posterior cornea with a more negative central power at the early postoperative stage. With wound remodelling, hypercellular fibrotic stromal scars at the flap wound margins develop, these biomechanical changes resulted in greater corneal stability, allowing the posterior cornea to revert to its preoperative state. With regards to the 
posterior corneal astigmatism, there were no changes in either the vector components. No evidence of myopic regression or progressive corneal ectasia was observed during our 3 years of follow-up. Similar to Scheimpflug photography, SS-OCT can directly evaluate the corneal topography from the posterior corneal surface. Evaluating the posterior corneal elevation is of particular importance for early detection and monitoring of post-LASIK ectasia. A shorter scanning duration is essential to reduce motion artefacts, while a longer wavelength permits better light penetration with less scattering through the LASIK flap. We believe that due to the shorter scan duration (0.3 vs. 2 seconds) and longer wavelength of light source (1310 vs. $475 \mathrm{~nm}$ ) in SS-OCT than Scheimpflug imaging, the former provides a more precise and accurate assessment of the posterior corneal surface. Further studies should evaluate posterior corneal elevation at various optical zones to ascertain the stability of the posterior corneal surface demonstrated in this SS-OCT study.

\section{REFERENCES}

1. Randleman JB, Russell B, Ward MA, Thompson KP, Stulting RD. Risk factors and prognosis for corneal ectasia after LASIK. Ophthalmology 2003; 110: 267-75.

2. Chan TC, Biswas S, Yu M, Jhanji V. Longitudinal Evaluation of Cornea With Swept-Source Optical Coherence Tomography and Scheimpflug Imaging Before and After Lasik. Medicine (Baltimore) 2015; 94: e1219.

3. Chan TC, Liu D, Yu M, Jhanji V. Longitudinal evaluation of posterior corneal elevation after laser refractive surgery using swept-source optical coherence tomography. Ophthalmology 2015; 122: 687-92. 
4. Thibos LN, Wheeler W, Horner D. Power vectors: an application of Fourier analysis to the description and statistical analysis of refractive error. Optom Vis Sci 1997; 74: 367-75.

5. Dawson DG, Kramer TR, Grossniklaus HE, Waring GO, 3rd, Edelhauser HF. Histologic, ultrastructural, and immunofluorescent evaluation of human laserassisted in situ keratomileusis corneal wounds. Arch Ophthalmo/2005; 123: 741-56.

This article is protected by copyright. All rights reserved. 
Table 1: Refractive measurements, corneal keratometric and topographic measurements before and after femtosecond laser-assisted LASIK

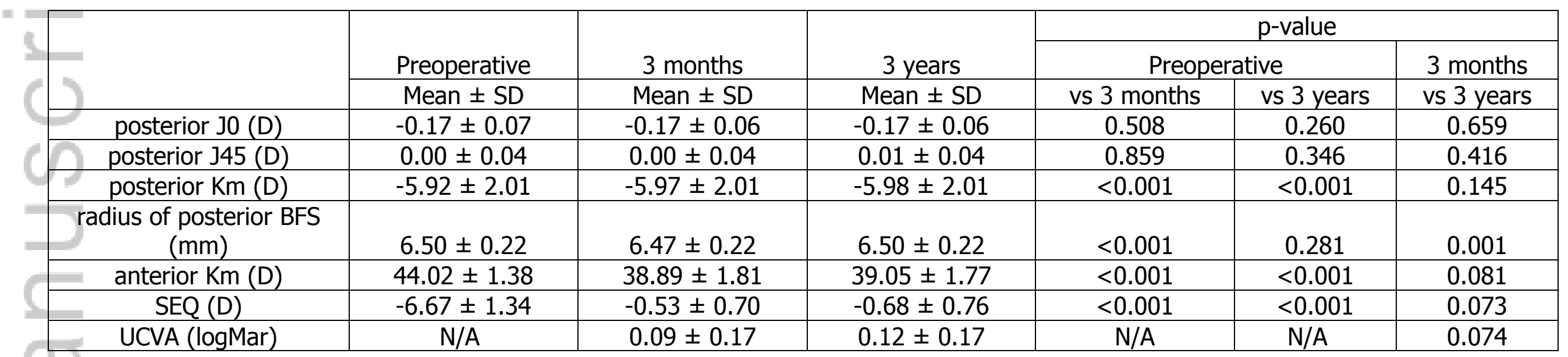

$\mathrm{Km}=$ mean keratometry; $\mathrm{BFS}=$ best fit sphere; $\mathrm{SEQ}=$ spherical equivalent; $\mathrm{SD}=$ standard deviation; $\mathrm{D}=$ diopter; $\mathrm{mm}$

$=$ millimeter, $\mathrm{N} / \mathrm{A}=$ not available 


\section{University Library}

\section{- M M N E R VA A gateway to Melbourne's research publications}

Minerva Access is the Institutional Repository of The University of Melbourne

Author/s:

Wan, KH;Chan, TCY;Yu, M;Jhanji, V

Title:

Longitudinal evaluation of posterior corneal changes after laser in situ keratomileusis in high myopia: a swept-source optical coherence tomography study

Date:

2018-09-01

\section{Citation:}

Wan, K. H., Chan, T. C. Y., Yu, M. \& Jhanji, V. (2018). Longitudinal evaluation of posterior corneal changes after laser in situ keratomileusis in high myopia: a swept-source optical coherence tomography study. CLINICAL AND EXPERIMENTAL OPHTHALMOLOGY, 46 (7), pp.824-826. https://doi.org/10.1111/ceo.13178.

Persistent Link:

http://hdl.handle.net/11343/283645 